\title{
On Decoupling of Quasi-Orthogonal Space-Time Block Codes based on Inherent Structure
}

\author{
Vasilios M. Kapinas and George K. Karagiannidis \\ Department of Electrical and \\ Computer Engineering \\ Aristotle University of Thessaloniki \\ Thessaloniki, Greece \\ Email: \{kapinas, geokarag\}@auth.gr
}

\begin{abstract}
In this paper we propose a linear decoupling scheme for multiple-input multiple-output (MIMO) antenna systems where spatial diversity is exploited through complex orthogonal (COD) or quasi-orthogonal (CQOD) designs. The proposed scheme is based on rewriting the space-time block code (ST BC) in an alternative form via generalized permutation matrices (GPM) and transforming the original system to an equivalent one, where all the transmitted symbols are decoupled, allowing thus a single symbol or symbol pair-wise maximum likelihood decoding (MLD) through a closed form expression (CFE).

Index Terms - Space-time block coding, quasi-orthogonal designs, maximum-likelihood decoding, generalized permutation matrices, wireless communications.
\end{abstract}

\section{INTRODUCTION}

Space-time block codes (STBC) exploit spatial diversity in multiple transmit antenna wireless systems. Orthogonal STBC (OSTBC) have been proved a highly attractive mean of combating fading by providing full-diversity with simple linear processing at the receiver. Quasi-OSTBC (QOSTBC) extended the family of orthogonal designs used in multiple-input multiple-output (MIMO) systems, achieving higher transmission rates for more than two transmit antennas at the expense of decoupling complexity, concerning complex symbol transmission. Thus, the issue of simple decoupling strategies of the transmitted symbols at the receiver has become a matter of great interest in recent research.

In [1]-[3] the authors introduced full-diversity space-time block codes for two, three and four transmit antennas achieving rates $1,1 / 2$ and $3 / 4$ respectively, giving at the same time maximum likelihood decoding (MLD) algorithms based only on linear processing at the receiver. A squaring method for simplifying the decoding of generalized complex orthogonal designs that were established in [4] with arbitrary number of transmit and receive antennas, was proposed in [5]. The need for STBC involving transmission of complex symbols with higher rates in multiple transmit antenna systems led to the ABBA code introduced in [6] and QOSTBC presented in [7] with decoding algorithms based on iterative interference cancellation and joint detection of symbol pairs correspondingly. In [8], [9] a decoding method for the code proposed in [7] for four transmit antennas was presented, based on channel matched filtering, leading therefore to two equivalent subsystems at the receiver part, providing distinct MLD for each one. Matched filtering with particular noise whitening was presented in [10], concerning decoding of QOSTBC that satisfy the construction criteria defined in [11]. In order to address the issue of decoding complexity of QOSTBC, the authors in [12] introduced a fast MLD algorithm that decomposes the original system into parallel subsystems followed by a sphere decoder for each subsystem and the maximal ratio combining (MRC) technique for combining the decouplers' outputs from all receiving branches. In [13], a unified mathematical expression for the decoding of generalized linear complex orthogonal designs was extracted, leading to a maximum likelihood (ML) single symbol based decision rule. General linear transformations of information symbols for construction of QOSTBC were studied in [14], that can provide pair-wise real symbol MLD whereas in [15] a low complexity $\mathrm{ML}$ decoder was proposed for QOSTBC employing four transmit antennas. A practical method for single symbol or symbol group decoding was mentioned in [16] for the codes proposed in the same manuscript. Finally, a pair-wise real symbol decoding was presented in [17] for ABBA QOSTBC, whereas in [18] the authors gave an efficient MIMO detection algorithm for codes being extensions of the Alamouti scheme.

Almost all decoding methods described above, presuppose the evaluation of the equivalent channel matrix that is characteristic of the structure body of the considered STBC. However, they don't give insights in its systematic construction, apart from those techniques concerning decoding of specific STBC structures. An exception is [13], where a general closed form decoding expression for STBC that can be written in linear dispersion (LD) matrices form, was derived.

In this paper, a unified method for transforming the original system into a linear decoupling scheme, suitable for $\mathrm{ML}$ single symbol or pair-wise symbol detection is described, regarding the majority of STBC derived from complex orthogonal (COD) or quasi-orthogonal (CQOD) designs. A different approach from [13] is considered here, in order to exploit the inherent structure of the transmit matrix. Specifically, we make use of the theory of generalized permutation matrices (GPM), concluding thus to a general closed form expression (CFE) 
for the equivalent channel matrix and the linear decoupling scheme. MLD can directly be achieved by minimizing the corresponding squared Euclidean distances between the linear processed received signals and all possible values of the transmitted symbols. The application of the proposed method to a sample of well-known STBC reveals its effectiveness, while simple matrix manipulations are required.

This paper is organized as follows. Section II establishes the notation adopted in this manuscript and describes the system model. Section III proposes the new linear decoupling scheme. In Section IV, we illustrate our decoupling method through some examples, while concluding remarks are given in Section $\mathrm{V}$.

\section{PRELiminaries}

\section{A. Notations}

- $\mathbf{Z} \in \mathbb{C}^{m \times n}$ defines a complex $m \times n$ matrix with elements $\left\{\begin{array}{ll}z_{i j} & \begin{array}{l}i=1,2, \ldots, m \\ j=1,2, \ldots, n\end{array}\end{array}\right\} \in \mathbb{C}$.

- $[\cdot]^{*},[\cdot]^{T},[\cdot]^{H}$ denote the conjugate, transpose and conjugate transpose of a matrix correspondingly.

- If $\mathbf{Z}_{i} \in \mathbb{C}^{p \times q},(i=1,2, \ldots, m)$ then $\operatorname{diag}\left(\mathbf{Z}_{i}\right)_{m}=$ $\operatorname{diag}\left(\mathbf{Z}_{1}, \mathbf{Z}_{2}, \ldots, \mathbf{Z}_{m}\right)$ denotes an $m p \times m q$ diagonal block matrix with elements $\left\{\begin{array}{ll}\delta_{i j} \mathbf{Z}_{i} \mid \begin{array}{l}i=1,2, \ldots, m \\ j=1,2, \ldots, m\end{array}\end{array}\right\} \in$ $\mathbb{C}^{p \times q}$ where $\delta_{i j}$ is the Kronecker delta.

- If $S=\{i: i \in \mathbb{N} ; i \leq m\}$ and $S_{i} \subseteq P(S)$, where $P(S)$ is the powerset of $S$, and $S_{i}^{c}$ is the complement of $S_{i}$ in $S$, then for the complex matrix $\mathbf{Z} \in \mathbb{C}^{m \times n}$ we define the following operator:

$$
\Lambda_{S_{i}}(\mathbf{Z}) \triangleq\left\{z_{i j}^{*}\left|\begin{array}{l}
i \in S_{i} \\
j=1,2, \ldots, n
\end{array} ; z_{i j}\right| \begin{array}{l}
i \in S_{i}^{c} \\
j=1,2, \ldots, n
\end{array}\right\} .
$$

Obviously, the action of this operator on $\mathbf{Z}$ gives the same matrix but with those rows included in the subset $S_{i}$ conjugated. We have to note that in this manuscript, operator $\Lambda_{S_{i}}(\cdot)$ is applied to block matrices as well.

Useful Properties of $\Lambda_{S_{i}}(\cdot)$ :

The following properties of $\Lambda_{S_{i}}(\cdot)$ derive straightforwardly from its definition:

1) If $\mathbf{A}, \mathbf{B} \in \mathbb{C}^{m \times n}$ then $\Lambda_{S_{i}}(\mathbf{A}+\mathbf{B})=\Lambda_{S_{i}}(\mathbf{A})+$ $\Lambda_{S_{i}}(\mathbf{B})$.

2) If $\mathbf{G} \in \mathbb{R}^{m \times n}$ then $\Lambda_{S_{i}}(\mathbf{G})=\mathbf{G}$.

3) If $\mathbf{A} \in \mathbb{C}^{m \times n}$ and $\mathbf{L}_{1} \in \mathbb{R}^{n \times q}, \mathbf{L}_{2} \in \mathbb{R}^{p \times m}$ then $\Lambda_{S_{i}}\left(\mathbf{A L}_{1}\right)=\Lambda_{S_{i}}(\mathbf{A}) \mathbf{L}_{1}$ and $\Lambda_{S_{i}}\left(\mathbf{L}_{2} \mathbf{A}\right)=$ $\mathbf{L}_{2} \Lambda_{S_{i}}(\mathbf{A})$.

4) If $\mathbf{A}, \mathbf{B}$ are diagonal matrices, then $\Lambda_{S_{i}}(\mathbf{A B})=$ $\Lambda_{S_{i}}(\mathbf{A}) \Lambda_{S_{i}}(\mathbf{B})$.

5) If $\mathbf{A} \in \mathbb{C}^{m \times n}$ then $\Lambda_{S_{i}}\left(\Lambda_{S_{i}}(\mathbf{A})\right)=\Lambda_{S_{i}}^{2}(\mathbf{A})=\mathbf{A}$.

6) If $\mathbf{A} \in \mathbb{C}^{m \times n}$ then $\left(\Lambda_{S_{i}}(\mathbf{A})\right)^{H}=\Lambda_{S_{i}}\left(\mathbf{A}^{H}\right)$.

\section{B. System Model}

We consider a MIMO system equipped with $N_{T}$ antennas at the transmitter and $N_{R}$ antennas at the receiver. Suppose the information data at the input of the encoder is represented by a set of $k$ symbols $\left\{s_{1}, s_{2}, \cdots, s_{k}\right\}$. After the modulation, a row vector $\mathbf{x}_{c}=\left[\begin{array}{llll}x_{1} & x_{2} & \cdots & x_{k}\end{array}\right]$ consisting of constellation symbols is mapped into the transmit matrix (or code matrix) $\mathrm{X} \in \mathbb{C}^{N \times N_{T}}$, where $N$ is the block length (number of time slots). Therefore, the rate $R$ of the space-time code is defined by $R=k / N$.

The data transmission is considered over a quasi-static channel and the channel gain from transmit antenna $i$ to receive antenna $j$ is denoted as $h_{i j}$. Hence, the entries of the channel matrix $\mathbf{H} \in \mathbb{C}^{N_{T}} \times N_{R}$ remain constant over a block of $N$ time slots and change independently from one block to another. The channel gains experience independent and identically distributed (i.i.d.) flat fading for all pairs $(i, j)$.

The received matrix $\mathbf{R} \in \mathbb{C}^{N \times N_{R}}$ can be modeled as

$$
\mathbf{R}=\mathbf{X H}+\mathbf{N},
$$

where $\mathbf{N} \in \mathbb{C}^{N \times N_{R}}$ is an additive white Gaussian noise (AWGN) matrix with entries $n_{i j}$ modeled as independent samples of zero-mean complex Gaussian random variables with variance $N_{\circ} / 2$ per real dimension. It is further assumed that the transmitter has no channel state information (CSI), whereas the receiver has perfect CSI.

\section{The Decoupling Scheme}

\section{A. Code matrix and GPM}

We focus on STBC where at each time-slot all $k$ symbols are transmitted via $N_{T}=k$ different transmit antennas. Hence, all rows of the code matrix $\mathbf{X}$ can be considered as permutations of either $\mathbf{x}_{c}$ or $\mathbf{x}_{c}^{*}$ entries, since many complex orthogonal or quasi-orthogonal STBC have one or more of their rows conjugated including both signed and unsigned entries of $\mathbf{x}_{C}$. Recalling from linear algebra that a GPM $\mathbf{G}$ is defined as a product of a nonsingular diagonal matrix $\mathbf{D}$ and a permutation matrix $\mathbf{P}$, the code matrix $\mathbf{X}$ can be written as

$$
\mathbf{X}=\left[\begin{array}{c}
\mathbf{x}_{c_{1}} \mathbf{G}_{1} \\
\vdots \\
\mathbf{x}_{c_{i}} \mathbf{G}_{i} \\
\vdots \\
\mathbf{x}_{c_{N}} \mathbf{G}_{N}
\end{array}\right],
$$

where $\mathbf{G}_{i} \in \mathbb{R}^{k \times k}, i=1,2, \ldots, N$ are trinary GPM of order $k$ with entries within the set $\{0,1,-1\}$. The $1 \times k$ row vectors $\mathbf{x}_{c_{i}} i=1,2, \ldots, N$ can be either $\mathbf{x}_{c}$ or $\mathbf{x}_{c}^{*}$, depending on their index. Specifically, if $S=\{i: i \in \mathbb{N} ; i \leq N\}$ and $S_{i} \subseteq S$ is the set of $\mathbf{X}$ rows involving the entries of $\mathbf{x}_{c}^{*}$, then we can rewrite $\mathbf{X}$ in the following form

$$
\begin{aligned}
\mathbf{X} & =\left[\begin{array}{c}
\mathbf{x}_{c_{1}} \mathbf{G}_{\mathbf{1}} \\
\vdots \\
\mathbf{x}_{C_{S_{i}}} \mathbf{G}_{S_{i}} \\
\vdots \\
\mathbf{x}_{c_{N}} \mathbf{G}_{N}
\end{array}\right]=\left[\begin{array}{ccccc}
\mathbf{x}_{c_{1}} & \mathbf{0} & \mathbf{0} & \mathbf{0} & \mathbf{0} \\
\mathbf{0} & \ddots & \mathbf{0} & \mathbf{0} & \mathbf{0} \\
\mathbf{0} & \mathbf{0} & \mathbf{x}_{C_{S_{i}}} & \mathbf{0} & \mathbf{0} \\
\mathbf{0} & \mathbf{0} & \mathbf{0} & \ddots & \mathbf{0} \\
\mathbf{0} & \mathbf{0} & \mathbf{0} & \mathbf{0} & \mathbf{x}_{C_{N}}
\end{array}\right]\left[\begin{array}{c}
\mathbf{G}_{1} \\
\vdots \\
\mathbf{G}_{S_{i}} \\
\vdots \\
\mathbf{G}_{N}
\end{array}\right] \\
& =\operatorname{diag}\left(\mathbf{x}_{c_{i}}\right)_{N} \dot{\mathbf{G}}
\end{aligned}
$$

where we have substituted the row indices of $\mathbf{X}$ included in $S_{i}$ with $S_{i}$ index notation, in the sense that for these 
indices $\mathbf{x}_{C_{S_{i}}}=\mathrm{x}_{c}^{*}$ whereas for all the others included in the complement set $S_{i}^{c}=S-S_{i}$ it holds that $\mathbf{x}_{C_{S_{i}^{o}}}=\mathbf{x}_{c}$. The $N k \times k$ real matrix $\dot{\mathbf{G}}$ depends on the general distribution of $\left\{ \pm x_{1}, \pm x_{2}, \cdots, \pm x_{k}\right\}$ entries in the structure body of the code. We can continue by using the operator $\Lambda_{S_{i}}(\cdot)$ determined in Section II

$$
\begin{aligned}
\mathbf{X} & =\left[\begin{array}{ccccc}
\mathbf{x}_{c} & \mathbf{0} & \mathbf{0} & \mathbf{0} & \mathbf{0} \\
\mathbf{0} & \ddots & \mathbf{0} & \mathbf{0} & \mathbf{0} \\
\mathbf{0} & \mathbf{0} & \mathbf{x}_{c}^{*} & \mathbf{0} & \mathbf{0} \\
\mathbf{0} & \mathbf{0} & \mathbf{0} & \ddots & \mathbf{0} \\
\mathbf{0} & \mathbf{0} & \mathbf{0} & \mathbf{0} & \mathbf{x}_{c}
\end{array}\right] \dot{\mathbf{G}} \\
& =\Lambda_{S_{i}}\left(\left[\begin{array}{ccc}
\mathbf{x}_{c} & \mathbf{0} & \mathbf{0} \\
\mathbf{0} & \ddots & \mathbf{0} \\
\mathbf{0} & \mathbf{0} & \mathbf{x}_{c}
\end{array}\right]\right) \dot{\mathbf{G}} \\
& =\Lambda_{S_{i}}\left(\operatorname{diag}\left(\mathbf{x}_{c}\right)_{N}\right) \dot{\mathbf{G}} .
\end{aligned}
$$

\section{B. Predecoding}

The alternative forms of the code matrix $\mathbf{X}$ derived above, can help us to manipulate the equation describing the system model in (1), so as to extract an equivalent system model suitable for $\mathrm{ML}$ decoding. To do this, we first expand (1) in element-wise form

$$
\begin{aligned}
& {\left[\begin{array}{ccc}
r_{11} & \cdots & r_{1 N_{r}} \\
\vdots & \ddots & \vdots \\
r_{N 1} & \cdots & r_{N N_{r}}
\end{array}\right] } \\
= & \mathbf{X}\left[\begin{array}{ccc}
h_{11} & \cdots & h_{1 N_{r}} \\
\vdots & \ddots & \vdots \\
h_{N 1} & \cdots & h_{N N_{r}}
\end{array}\right]+\left[\begin{array}{ccc}
n_{11} & \cdots & n_{1 N_{r}} \\
\vdots & \ddots & \vdots \\
n_{N 1} & \cdots & n_{N N_{r}}
\end{array}\right] .
\end{aligned}
$$

For the sake of simplicity, we will continue on the predecoding scheme examining each receiving antenna separately. Consequently, the subsystem concerning $j^{\text {th }}$ receiving antenna $\left(j=1,2, \ldots, N_{r}\right)$ is

$$
\mathbf{r}_{j}=\mathbf{X h}_{j}+\mathbf{n}_{j} .
$$

We apply $\Lambda_{S_{i}}(\cdot)$ operator on both sides of (5) considering (3) and the properties presented in Section II

$$
\begin{aligned}
\Lambda_{S_{i}}\left(\mathbf{r}_{j}\right)= & \Lambda_{S_{i}}\left(\mathbf{X h}_{j}+\mathbf{n}_{j}\right) \\
= & \Lambda_{S_{i}}\left(\left[\begin{array}{c}
\mathbf{x}_{c_{1}} \mathbf{G}_{1} \mathbf{h}_{j} \\
\vdots \\
\mathbf{x}_{\mathcal{C}_{i}} \mathbf{G}_{S_{i}} \mathbf{h}_{j} \\
\vdots \\
\mathbf{x}_{C_{N}} \mathbf{G}_{N} \mathbf{h}_{j}
\end{array}\right]\right)+\Lambda_{S_{i}}\left(\mathbf{n}_{j}\right) .
\end{aligned}
$$

Having into mind that $\Lambda_{S_{i}}(\cdot)$ conjugates those rows with indices being included in $S_{i}$, we continue with the following matrix manipulations

$$
\begin{aligned}
\Lambda_{S_{i}}\left(\mathbf{r}_{j}\right)= & {\left[\begin{array}{lllll}
\mathbf{x}_{c} \mathbf{G}_{1} \mathbf{h}_{j} & \cdots & \mathbf{x}_{c} \mathbf{G}_{S_{i}} \mathbf{h}_{j}^{*} & \cdots & \mathbf{x}_{c} \mathbf{G}_{N} \mathbf{h}_{j}
\end{array}\right]^{T} } \\
& +\Lambda_{S_{i}}\left(\mathbf{n}_{j}\right)
\end{aligned}
$$

where we considered $\left(\mathbf{x}_{c} \mathbf{G}_{i} \mathbf{h}_{j}\right)^{T}=\mathbf{x}_{c} \mathbf{G}_{i} \mathbf{h}_{j}$ since each entry $\left(\mathbf{x}_{c} \mathbf{G}_{i} \mathbf{h}_{j}\right)_{i=1,2, \ldots, N}$ is a complex number rather than a submatrix. Therefore, it holds that

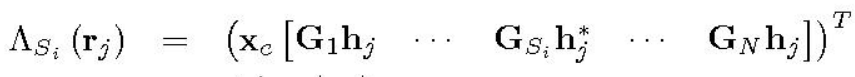

$$
\begin{aligned}
& +\Lambda_{S_{i}}\left(\mathbf{n}_{j}\right) \\
& =\left[\begin{array}{lllll}
\mathbf{G}_{1} \mathbf{h}_{j} & \cdots & \mathbf{G}_{S_{i}} \mathbf{h}_{j}^{*} & \cdots & \mathbf{G}_{N} \mathbf{h}_{j}
\end{array}\right]^{T} \mathbf{x}_{c}^{T} \\
& +\Lambda_{S_{i}}\left(\mathbf{n}_{j}\right)
\end{aligned}
$$

or

$$
\Lambda_{S_{i}}\left(\mathbf{r}_{j}\right)=\dot{\mathbf{H}} \mathbf{x}_{c}^{T}+\Lambda_{S_{i}}\left(\mathbf{n}_{j}\right) .
$$

Obviously, the structure and properties of $\dot{\mathbf{H}}$ are of great importance since they affect the decoupling of the equivalent scheme. For this reason, we will analyze it further

$$
\begin{aligned}
\dot{\mathbf{H}} & \left.=\left(\begin{array}{llllll}
{\left[\mathbf{G}_{1}\right.} & \cdots & \mathbf{G}_{S_{i}} & \cdots & \mathbf{G}_{N}
\end{array}\right] \Lambda_{S_{i}}\left(\operatorname{diag}\left(\mathbf{h}_{j}\right)_{N}\right)\right)^{T} \\
& =\Lambda_{S_{i}}\left(\operatorname{diag}\left(\mathbf{h}_{j}^{T}\right)_{N}\right)\left[\begin{array}{lllll}
\mathbf{G}_{1} & \cdots & \mathbf{G}_{S_{i}} & \cdots & \mathbf{G}_{N}
\end{array}\right]^{T} \\
& =\Lambda_{S_{i}}\left(\operatorname{diag}\left(\mathbf{h}_{j}^{T}\right)_{N}\right) \ddot{\mathbf{G}}^{T},
\end{aligned}
$$

with $\ddot{\mathrm{G}}$ the $k \times N k$ appended matrix of all the GPM related to the construction pattern of the code matrix $\mathbf{X}$ shown in (2). Let us now compare the structure of the two matrices $\mathbf{X}$ and $\dot{\mathbf{H}}$, as these derived in (4) and (7)

$$
\mathbf{X}=\Lambda_{S_{i}}\left(\operatorname{diag}\left(\mathbf{x}_{c}\right)_{N}\right)\left[\begin{array}{c}
\mathbf{G}_{1} \\
\vdots \\
\mathbf{G}_{S_{i}} \\
\vdots \\
\mathbf{G}_{N}
\end{array}\right]
$$

and

$$
\dot{\mathbf{H}}=\Lambda_{S_{i}}\left(\operatorname{diag}\left(\mathbf{h}_{j}^{T}\right)_{N}\right)\left[\begin{array}{c}
\mathbf{G}_{1}^{T} \\
\vdots \\
\mathbf{G}_{S_{i}}^{T} \\
\vdots \\
\mathbf{G}_{N}^{T}
\end{array}\right] .
$$

As mentioned above, the GPM $\left(\mathbf{G}_{i}\right)_{i=1,2, \ldots, N}$ can be written as $\mathbf{G}_{i}=\mathbf{D}_{i} \mathbf{P}_{i}$, where $\mathbf{P}_{i} k \times k$ permutation matrices satisfying orthogonality $\mathbf{P}_{i} \mathbf{P}_{i}^{T}=\mathbf{P}_{i}^{T} \mathbf{P}_{i}=\mathbf{I}_{k}$ [19] and $\mathbf{D}_{i}$ nonsingular diagonal $k \times k$ matrices with diagonal elements included in our case in the set $\{1,-1\}$. Therefore, $\quad \mathbf{D}_{i} * \mathbf{D}_{i}^{T}=\operatorname{diag}(\{ \pm 1\})_{k} \operatorname{diag}(\{ \pm 1\})_{k}^{T}=$ $\operatorname{diag}\left(\left\{( \pm 1)^{2}\right\}\right)_{k}=\operatorname{diag}(1)_{k}=\mathbf{I}_{k}$ (in the same way $\left.\mathbf{D}_{i}^{T} * \mathbf{D}_{i}=\mathbf{I}_{k}\right)$ and $\mathbf{G}_{i} \mathbf{G}_{i}^{T}=\mathbf{D}_{i} \mathbf{P}_{i}\left(\mathbf{D}_{i} \mathbf{P}_{i}\right)^{T}=$ $\mathbf{D}_{i} \mathbf{P}_{i} \mathbf{P}_{i}^{T} \mathbf{D}_{i}^{T}=\mathbf{D}_{i} * \mathbf{D}_{i}^{T}=\mathbf{I}_{k}$ (in the same way $\mathbf{G}_{i}^{T} \mathbf{G}_{i}=$ $\mathbf{I}_{k}$ ). Consequently, $\left(\mathbf{G}_{i}\right)_{i=1,2, \ldots, N}$ and $\left(\mathbf{G}_{i}^{T}\right)_{i=1,2, \ldots, N}$ are orthogonal matrices. This realization gives evidence that $\mathbf{X}$ and $\dot{\mathbf{H}}$ obtain similar patterns. Hence, given that $\mathbf{X}$ is a QOSTBC, it follows that $\dot{\mathbf{H}}$ stems from a quasi-orthogonal design (QOD) as well. Thus, the product $\dot{\mathbf{H}}^{H} \dot{\mathbf{H}}$ gives a matrix that according to QOD theory can be rearranged into a block diagonal matrix. In the same way, if $\mathbf{X}$ is orthogonal then $\dot{\mathbf{H}}^{H} \dot{\mathbf{H}}$ gives a scaled identity matrix. Therefore, the next linear 
processing step for the receiver is to apply a matched filter $\mathbf{H}_{R}^{j}=\dot{\mathbf{H}^{H}}=\ddot{\mathbf{G}} \Lambda_{S_{i}}\left(\operatorname{diag}\left(\mathbf{h}_{j}^{*}\right)_{N}\right)$ to the signal $\Lambda_{S_{i}}\left(\mathbf{r}_{j}\right)$ shown in (6), in order to separate groups of symbols (or single symbols in the orthogonal case) for $\mathrm{ML}$ joint decoding

$$
\begin{aligned}
\mathbf{H}_{R}^{j} \Lambda_{S_{i}}\left(\mathbf{r}_{j}\right) & =\left(\mathbf{H}_{R}^{j} \dot{\mathbf{H}}\right) \mathbf{x}_{c}^{T}+\mathbf{H}_{R}^{j} \Lambda_{S_{i}}\left(\mathbf{n}_{j}\right) \\
& =\overline{\mathbf{G}}_{j} \mathbf{x}_{c}^{T}+\mathbf{H}_{R^{j}}^{j} \Lambda_{S_{i}}\left(\mathbf{n}_{j}\right),
\end{aligned}
$$

with $\overline{\mathbf{G}}_{j}$ being

$$
\begin{aligned}
\overline{\mathbf{G}}_{j} & =\ddot{\mathbf{G}} \Lambda_{S_{i}}\left(\operatorname{diag}\left(\mathbf{h}_{j}^{*}\right)_{N}\right) \Lambda_{S_{i}}\left(\operatorname{diag}\left(\mathbf{h}_{j}^{T}\right)_{N}\right) \ddot{\mathbf{G}}^{T}(9) \\
& =\ddot{\mathbf{G}} \Lambda_{S_{i}}\left(\operatorname{diag}\left(\mathbf{h}_{j}^{*} \mathbf{h}_{j}^{T}\right)_{N}\right) \ddot{\mathbf{G}}^{T} .
\end{aligned}
$$

Equation (8) represents the equivalent linear decoupling scheme concerning $j^{\text {th }}$ receiving antenna, written as $\overline{\mathbf{r}}_{j}=$ $\overline{\mathbf{G}}_{j} \mathbf{x}_{c}^{T}+\overline{\mathbf{n}}_{j}$ with $\overline{\mathbf{r}}_{j}=\mathbf{H}_{R}^{j} \Lambda_{S_{i}}\left(\mathbf{r}_{j}\right)$ and $\overline{\mathbf{n}}_{j}=\mathbf{H}_{R}^{j} \Lambda_{S_{i}}\left(\mathbf{n}_{j}\right)$ where $\mathbf{H}_{R}^{j}$ is the conjugate transpose of the equivalent channel matrix concerning $j^{\text {th }}$ receiving antenna. Now, if we take into consideration the rest of receiving branches as well, then the linear processing is completed by just superimposing all the vectors $\overline{\mathbf{r}}_{j}$ associated with them. Thus, the CFE describing the system model after predecoder is given by

$$
\overline{\mathbf{R}}=\sum_{j=1}^{N_{r}} \overline{\mathbf{r}}_{j}=\sum_{j=1}^{N_{r}} \overline{\mathbf{G}}_{j} \mathbf{x}_{c}^{T}+\sum_{j=1}^{N_{r}} \overline{\mathbf{n}}_{j}=\overline{\mathbf{G}} \mathbf{x}_{c}^{T}+\overline{\mathbf{N}},
$$

with $\overline{\mathbf{G}}$ the $k \times k$ matrix related to the diversity gain achieved by the specific STBC, given as

$$
\overline{\mathbf{G}}=\ddot{\mathbf{G}} \Lambda_{S_{i}}\left(\operatorname{diag}\left(\sum_{j=1}^{N_{r}} \mathbf{h}_{j}^{*} \mathbf{h}_{j}^{T}\right)_{N}\right) \ddot{\mathbf{G}}^{T} .
$$

The linear processing required at the receiver for achieving single symbol or symbol pair-wise $\mathrm{ML}$ decoding is

$$
\overline{\mathbf{R}}=\sum_{j=1}^{N_{r}} \mathbf{H}_{R^{j}}^{j} \Lambda_{S_{i}}\left(\mathbf{r}_{j}\right)=\ddot{\mathbf{G}} \sum_{j=1}^{N_{r}} \Lambda_{S_{i}}\left(\operatorname{diag}\left(\mathbf{h}_{j}^{*}\right)_{N}\right) \Lambda_{S_{i}}\left(\mathbf{r}_{j}\right) .
$$

\section{Applications}

In the following indicative examples, three typical cases of STBC, including both orthogonal and quasi-orthogonal block codes, are decoupled. For the sake of simplicity, we will consider multiple receive antennas $\left(N_{R}=2\right)$ only in the first example (Alamouti case).

\section{A. Alamouti [1] - Rate 1}

The constellation vector $\mathbf{x}_{c}=\left[\begin{array}{ll}x_{1} & x_{2}\end{array}\right]$, is transmitted via two antennas $\left(N_{T}=2\right)$ in a frame of two time-slots $(N=2)$. The received signal vector, considering two receive antennas $\left(N_{R}=2\right)$, is equal to

$$
\left[\begin{array}{ll}
r_{11} & r_{12} \\
r_{21} & r_{22}
\end{array}\right]=\underbrace{\left[\begin{array}{rr}
x_{1} & x_{2} \\
-x_{2}^{*} & x_{1}^{*}
\end{array}\right]}_{\mathbf{X}_{A L}}\left[\begin{array}{ll}
h_{11} & h_{12} \\
h_{21} & h_{22}
\end{array}\right]+\left[\begin{array}{ll}
n_{11} & n_{12} \\
n_{21} & n_{22}
\end{array}\right] .
$$

The related GPM to the structure of code matrix $\mathbf{X}_{A L}$ are

$$
\mathbf{G}_{1}^{A L}=\left[\begin{array}{ll}
1 & 0 \\
0 & 1
\end{array}\right], \mathbf{G}_{2}^{A L}=\left[\begin{array}{rr}
0 & 1 \\
-1 & 0
\end{array}\right] .
$$

Thus,

$$
\ddot{\mathbf{G}}_{A L}=\left[\begin{array}{ll}
\mathbf{G}_{1}^{A L} & \mathbf{G}_{2}^{A L}
\end{array}\right]=\left[\begin{array}{rrrr}
1 & 0 & 0 & 1 \\
0 & 1 & -1 & 0
\end{array}\right]
$$

and the output of the predecoder deriving from (12) is

$$
\begin{aligned}
\overline{\mathbf{R}}_{A L}= & \ddot{\mathbf{G}}_{A L} \Lambda_{S_{i}}\left(\operatorname{diag}\left(\mathbf{h}_{1}^{*}\right)_{N}\right) \Lambda_{S_{i}}\left(\mathbf{r}_{1}\right) \\
& +\ddot{\mathbf{G}}_{A L} \Lambda_{S_{i}}\left(\operatorname{diag}\left(\mathbf{h}_{2}^{*}\right)_{N}\right) \Lambda_{S_{i}}\left(\mathbf{r}_{2}\right) \\
= & \ddot{\mathbf{G}}_{A L}\left(\left[\begin{array}{cc}
h_{11}^{*} & 0 \\
h_{21}^{*} & 0 \\
0 & h_{11} \\
0 & h_{21}
\end{array}\right]\left[\begin{array}{l}
r_{11} \\
r_{21}^{*}
\end{array}\right]+\left[\begin{array}{cc}
h_{12}^{*} & 0 \\
h_{22}^{*} & 0 \\
0 & h_{12} \\
0 & h_{22}
\end{array}\right]\left[\begin{array}{l}
r_{12} \\
r_{22}^{*}
\end{array}\right]\right) \\
= & {\left[\begin{array}{l}
h_{11}^{*} r_{11}+h_{12}^{*} r_{12}+h_{21} r_{21}^{*}+h_{22} r_{22}^{*} \\
h_{21}^{*} r_{11}+h_{22}^{*} r_{12}-h_{11} r_{21}^{*}-h_{12} r_{22}^{*}
\end{array}\right] }
\end{aligned}
$$

where we considered $S_{i}=\{2\}$, since $\mathbf{X}_{A L}$ involves the conjugate entries of $\mathbf{x}_{c}$ in its second row. Furthermore, code matrix is orthogonal and therefore the symbols have to be completely decoupled after the linear processing at the receiver. Indeed, if we evaluate $\overline{\mathbf{G}}_{A L}$ matrix by using (11), we realize that is diagonal with form given by

$$
\overline{\mathbf{G}}_{A L}=\left[\begin{array}{cc}
a & 0 \\
0 & a
\end{array}\right], a=\left|h_{11}\right|^{2}+\left|h_{12}\right|^{2}+\left|h_{21}\right|^{2}+\left|h_{22}\right|^{2} .
$$

\section{B. Tirkkonen [6] - Rate 1}

In the ABBA code $\left(N_{T}=4, N_{R}=1\right)$

$$
\mathbf{X}_{A B B A}=\left[\begin{array}{rrrr}
x_{1} & x_{2} & x_{3} & x_{4} \\
-x_{2}^{*} & x_{1}^{*} & -x_{4}^{*} & x_{3}^{*} \\
x_{3} & x_{4} & x_{1} & x_{2} \\
-x_{4}^{*} & x_{3}^{*} & -x_{2}^{*} & x_{1}^{*}
\end{array}\right]
$$

its structural decoupling information can be expressed through $S_{i}=\{2,4\}$ and

$$
\begin{aligned}
\ddot{\mathbf{G}}_{A B B A}= & {\left[\begin{array}{rrrrrrrrr}
1 & 0 & 0 & 0 & 0 & 1 & 0 & 0 & \\
0 & 1 & 0 & 0 & -1 & 0 & 0 & 0 & \ldots \\
0 & 0 & 1 & 0 & 0 & 0 & 0 & 1 & \\
0 & 0 & 0 & 1 & 0 & 0 & -1 & 0 & \\
0 & 0 & 1 & 0 & 0 & 0 & 0 & 1 \\
& \cdots & 0 & 0 & 1 & 0 & 0 & -1 & 0 \\
1 & 0 & 0 & 0 & 0 & 1 & 0 & 0 \\
0 & 1 & 0 & 0 & -1 & 0 & 0 & 0
\end{array}\right] . }
\end{aligned}
$$

Hence, the predecoder output is

$$
\overline{\mathbf{R}}_{A B B A}=\left[\begin{array}{l}
h_{1}^{*} r_{1}+h_{2} r_{2}^{*}+h_{3}^{*} r_{3}+h_{4} r_{4}^{*} \\
h_{2}^{*} r_{1}-h_{1} r_{2}^{*}+h_{4}^{*} r_{3}-h_{3} r_{4}^{*} \\
h_{3}^{*} r_{1}+h_{4} r_{2}^{*}+h_{1}^{*} r_{3}+h_{2} r_{4}^{*} \\
h_{4}^{*} r_{1}-h_{3} r_{2}^{*}+h_{2}^{*} r_{3}-h_{1} r_{4}^{*}
\end{array}\right],
$$


given that $\mathbf{H}=\left[\begin{array}{llll}h_{1} & h_{2} & h_{3} & h_{4}\end{array}\right]^{T}$ is the channel matrix, whereas the decoupling matrix is given as

$\overline{\mathbf{G}}_{A B B A}=\left[\begin{array}{cccc}a & 0 & b & 0 \\ 0 & a & 0 & b \\ b & 0 & a & 0 \\ 0 & b & 0 & a\end{array}\right], \quad \begin{aligned} & a=\left|h_{1}\right|^{2}+\left|h_{2}\right|^{2}+\left|h_{3}\right|^{2}+\left|h_{4}\right|^{2} \\ & b=h_{1} h_{3}^{*}+h_{1}^{*} h_{3}+h_{2} h_{4}^{*}+h_{2}^{*} h_{4}\end{aligned}$

\section{Jafarkhani [7] - Rate 1}

For the Jafarkhani code $\left(N_{T}=4, N_{R}=1\right)$

$$
\mathbf{X}_{J}=\left[\begin{array}{rrrr}
x_{1} & x_{2} & x_{3} & x_{4} \\
-x_{2}^{*} & x_{1}^{*} & -x_{4}^{*} & x_{3}^{*} \\
-x_{3}^{*} & -x_{4}^{*} & x_{1}^{*} & x_{2}^{*} \\
x_{4} & -x_{3} & -x_{2} & x_{1}
\end{array}\right]
$$

the inherent decoupling information obtained in $\mathbf{X}_{J}$ is conveyed via $S_{i}=\{2,3\}$ and

$$
\begin{aligned}
& \ddot{\mathbf{G}}_{J}=\left[\begin{array}{rrrrrrrrr}
1 & 0 & 0 & 0 & 0 & 1 & 0 & 0 & \\
0 & 1 & 0 & 0 & -1 & 0 & 0 & 0 \\
0 & 0 & 1 & 0 & 0 & 0 & 0 & 1 & \ldots \\
0 & 0 & 0 & 1 & 0 & 0 & -1 & 0
\end{array} \ldots\right. \\
& \left.\begin{array}{rrrrrrrrr}
0 & 0 & 1 & 0 & 0 & 0 & 0 & 1 \\
0 & 0 & 0 & 1 & 0 & 0 & -1 & 0 \\
-1 & 0 & 0 & 0 & 0 & -1 & 0 & 0 \\
0 & -1 & 0 & 0 & 1 & 0 & 0 & 0
\end{array}\right] .
\end{aligned}
$$

The linear processing at the receiver part results in the following vector

$$
\overline{\mathbf{R}}_{J}=\left[\begin{array}{l}
h_{1}^{*} r_{1}+h_{2} r_{2}^{*}+h_{3} r_{3}^{*}+h_{4}^{*} r_{4} \\
h_{2}^{*} r_{1}-h_{1} r_{2}^{*}+h_{4} r_{3}^{*}-h_{3}^{*} r_{4} \\
h_{3}^{*} r_{1}+h_{4} r_{2}^{*}-h_{1} r_{3}^{*}-h_{2}^{*} r_{4} \\
h_{4}^{*} r_{1}-h_{3} r_{2}^{*}-h_{2} r_{3}^{*}+h_{1}^{*} r_{4}
\end{array}\right],
$$

given that $\mathbf{H}=\left[\begin{array}{llll}h_{1} & h_{2} & h_{3} & h_{4}\end{array}\right]^{T}$ is the channel matrix. Thus, symbol groups are decoupled according to the matrix

$$
\begin{aligned}
& \overline{\mathbf{G}}_{J}= {\left[\begin{array}{rrrr}
a & 0 & 0 & b \\
0 & a & -b & 0 \\
0 & -b & a & 0 \\
b & 0 & 0 & a
\end{array}\right], } \\
& a=\left|h_{1}\right|^{2}+\left|h_{2}\right|^{2}+\left|h_{3}\right|^{2}+\left|h_{4}\right|^{2} \\
& b=h_{1} h_{4}^{*}+h_{1}^{*} h_{4}-h_{2} h_{3}^{*}-h_{2}^{*} h_{3} \\
& \text { V. CONCLUSION }
\end{aligned}
$$

\section{CONCLUSION}

In this paper, we derived a CFE for the equivalent channel matrix and the linear decoupling scheme of a MIMO system that exploits spatial diversity via STBC constructed from $\mathrm{COD}$ and $\mathrm{CQOD}$, considering the requirements presented in Section III. For illustration, in Section IV we presented some application examples of the proposed method to three wellknown in the literature STBC. Nonetheless, we note that these codes constitute just a representative sample of all the STBC categories this method can be applied to. For instance, the proposed scheme can decouple successfully the QOSTBC codes $\mathbf{M}_{4}, \mathbf{M}_{5}$ and $\mathbf{M}_{6}$ presented in [20].

\section{ACKNOWLEDGMENT}

This work was performed within the framework of the Satellite Communications Network of Excellence (SatNex) project (IST-507052) and its Phase-II, SatNex-II (IST- 27393), funded by the European Commission (EC) under its FP6 program. The authors would like to thank Chr. Liontas and S. Michos for their useful advice.

\section{REFERENCES}

[1] S. M. Alamouti, "A simple transmit diversity technique for wireless communications," IEEE J. Select. Areas Commun., vol. 16, pp. 14511458 , Oct. 1998 .

[2] V. Tarokh, H. Jafarkhani, and A. R. Calderbank, "Space-time block coding for wireless communications: Performance results," IEEE $J$ Select. Areas Commun., vol. 17, pp. 451-460, Mar. 1999.

[3] G. Ganesan, and P. Stoica, "Space-time block codes: A maximum SNR approach," IEEE Trans. Inform. Theory, vol. 47, pp. 1650-1656, May 2001.

[4] V. Tarokh, H. Jafarkhani, and A. R. Calderbank, "Space-time block codes from orthogonal designs," IEEE Trans. Inform. Theory, vol. 45, pp. 1456-1467, July 1999.

[5] X. Li, T. L. Luo, G. Yue, and C. Yin, "A squaring method to simplify the decoding of orthogonal space-time block codes," IEEE Trans. Commun., vol. 49 , pp. $1700-1703$, Oct. 2001.

[6] O. Tirkkonen, A. Boariu, and A. Hottinen, "Minimal non-orthogonality rate 1 space-time block code for $3+$ Tx antennas," Proc. IEEE ISSSTA2000, vol 2, pp. 429-432, September 2000.

[7] H. Jafarkhani, "A quasi-orthogonal space-time block code," IEEE Trans. Commun., vol. 49, pp. 1-4, Jan. 2001.

[8] A. Sezgin, E. A. Jorswieck, and H. Boche, "Performance criteria analysis and further performance results for quasi-orthogonal space-time block codes," ISSPIT 2003, Darmstadt, Germany, pp. 345-348, December 14172003.

[9] A. Sezgin, and E. A. Jorswieck, "On optimal constellations for quasiorthogonal space-time codes," in Proceedings of the 2003 IEEE International Conference on Acoustics, Speech, and Signal Processing, ICASSP 2003, vol. 4, pp. 345-348, Hong-Kong, April 2003.

[10] C. Yuen, Y. L. Guan, and T. T. Tjhung, "Decoding of quasiorthogonal space-time block code with noise whitening" IEEE Pro. PIMRC 2003, vol. 3, pp. 2166-2170, Sept. 2003.

[11] C. Yuen, Y. L. Guan, and T. T. Tjhung, " Full-Rate Full-Diversity STBC with Constellation Rotation," Proc. VTC 2003, Spring vol. 1, pp. 296300, Seogwipo, Korea, April 2003.

[12] L. He, and H. Ge, "Fast maximum likelihood decoding of quasiorthogonal codes," Asilomar Conference on Signals, Systems, and Computers, 2003.

[13] C. Xu, and K. S. Kwak, "On decoding algorithm and performance of space-time block codes," IEEE Trans. on Wireless Commun., vol. 4, pp. $825-829$, May 2005.

[14] H. Wang, D. Wang, and X.-G. Xia, "On optimal quasi-orthogonal space-time block codes with minimum decoding complexity," Proc. International Symposium on Information Theory, ISIT 2005, pp. 11681172, 4-9 Sept. 2005.

[15] M. T. Le, V.-S. Pham, L. Mai, and G. Yoon, "Low-complexity maximum-likelihood decoder for four-transmit-antenna quasi-orthogonal space-time block code," IEEE Trans. Commun., vol. 53, pp. 1817-1821, Nov. 2005.

[16] L. Xian, and H. Liu, "Rate-one space-time block codes with full diversity," IEEE Trans. Commun., vol. 53, pp. 1986-1990, Dec. 2005.

[17] D. N. Dao, and C. Tellambura, "A general method to decode ABBA quasi-orthogonal space-time block codes," IEEE Commun. Lett., vol. 10 , pp. $713-715$, Oct. 2006.

[18] M. Arar, and A. Yongacoglu, "Efficient detection algorithm for $2 \mathrm{Nx} 2 \mathrm{~N}$ MIMO systems using alamouti code and $\mathrm{QR}$ decomposition," IEEE Commun. Lett., vol. 10, pp. 819-821, Dec. 2006.

[19] G. H. Golub, and C. F. Van Loan, Matrix Computations, The Johns Hopkins University Press, Baltimore, MD, $3^{\text {rd }}$ edition, 1996.

[20] Y. Zhao, and C. He, "Design and Performance Analysis of QuasiOrthogonal Space-Time Block Codes for Four Antennae," IEICE Trans. Fundam. Electron. Commun. Comput. Sci., vol. E88-A, Nov. 2005. 